\title{
Translating the Investigator's Static Global Assessment to the Eczema Area and Severity Index in Studies of Crisaborole for Atopic Dermatitis
}

\author{
Jacob P. Thyssen · Chuanbo Zang • Maureen P. Neary • \\ Andrew G. Bushmakin · Joseph C. Cappelleri · Amy Cha · \\ Christopher Russo • Thomas A. Luger
}

Received: December 23, 2020 / Accepted: February 25, 2021 / Published online: March 13, 2021

(C) The Author(s) 2021

\section{ABSTRACT}

Introduction: Atopic dermatitis (AD) severity was measured in two phase 3 US studies of crisaborole ointment, $2 \%$, in patients aged $\geq 2$ years using the Investigator's Static Global Assessment (ISGA), an FDA-recommended scale. Eczema Area and Severity Index (EASI) is a validated scale used globally to assess AD severity in clinical trials. The objective of this study is to aid interpretability of ISGA by translating ISGA scores to EASI scores.

Methods: ISGA was mapped to EASI using published EASI severity strata by Chopra et al. and Leshem et al. and pooled data from phase 3

Supplementary Information The online version contains supplementary material available at https:// doi.org/10.1007/s13555-021-00509-9.

J. P. Thyssen $(\bowtie)$

Department of Dermatology, Bispebjerg Hospital, University of Copenhagen, Copenhagen, Denmark e-mail: jacob.pontoppidan.thyssen@regionh.dk

C. Zang - M. P. Neary · C. Russo

Pfizer Inc., Collegeville, PA, USA

A. G. Bushmakin · J. C. Cappelleri

Pfizer Inc, Groton, CT, USA

A. Cha

Pfizer Inc., New York, NY, USA

T. A. Luger

Westphalian Wilhelms University of Münster,

Münster, Germany trials CrisADe CORE 1 and CORE 2, which evaluated crisaborole in patients aged $\geq 2$ years with mild-to-moderate AD (crisaborole, $n=1016$; vehicle, $n=506$ ). Least squares mean (LSM) percentage change from baseline (\%CFB) in EASI and proportion of patients with 50\%, 75\%, and 90\% improvement (EASI-50, EASI-75, and EASI-90, respectively) on day 29 were computed for mapped EASI. The relationship between changes in ISGA and changes in EASI was assessed using data from three abrocitinib trials.

Results: ISGA was mapped to EASI using 70,000 random simulations. LSM (standard error) for \%CFB in mapped EASI at day 29 (crisaborole versus vehicle) was $-26.3 \%$ (17) versus $45.2 \%$ (35) $(P=0.0671)$ using Chopra strata and $-43.1 \%$ (4.6) versus $-5.2 \% \quad(8.4) \quad(P<0.0001) \quad$ using Leshem strata. EASI-50, EASI-75, and EASI-90 rates were $72.1 \%$ versus $57.6 \%, 63.0 \%$ versus $47.8 \%$, and $55.0 \%$ versus $40.1 \%$, respectively, using Chopra strata $(P<0.0001$ for each difference). These rates were $68.8 \%$ versus $54.0 \%, 54.8 \%$ versus $40.5 \%$, and $38.9 \%$ versus $27.2 \%$, respectively $(P<0.0001$ for each difference) using Leshem strata. Mean two-point improvement in ISGA was comparable to EASI-90.

Conclusion: Mapped EASI results were consistent with ISGA results in crisaborole phase 3 trials. Simulation methodologies yielded consistent results and may aid in interpretability of ISGA across clinical studies. 
Trial Registration: ClinicalTrials.gov identifier: NCT02118766, NCT02118792.

Keywords: Atopic dermatitis; Clinical trial; Crisaborole; Eczema Area and Severity Index; Investigator's Static Global Assessment; Severity strata

\section{Key Summary Points}

Why carry out this study?

The Investigator's (Static) Global Assessment [I(S)GA] is recommended by the US Food and Drug Administration for assessment of the severity of atopic dermatitis (AD) in clinical trials, whereas the Eczema Area and Severity Index (EASI) is recommended by the Harmonizing Outcome Measures for Eczema initiative for the same purpose.

In phase 3 clinical trials of crisaborole ointment, $2 \%$, the primary outcome was assessed using the ISGA.

The objective of the present analysis was to translate ISGA scores to EASI scores using clinical trial data, thereby aiding in the interpretability of trial data that use ISGA-based endpoints.

\section{What was learned from this study?}

This study showed, through simulation analysis, the consistency of results obtained using mapped EASI scores across key clinical outcomes measures commonly reported in clinical trials in $\mathrm{AD}$ (i.e., \% change from baseline, EASI-50, EASI-75, EASI-90).

This analysis provides an approach that may be useful in other studies to further assist in interpretability of ISGA reported in clinical studies.

\section{DIGITAL FEATURES}

This article is published with digital features, including summary slide, to facilitate understanding of the article. To view digital features for this article go to https://doi.org/10.6084/ m9.figshare.14096269.

\section{INTRODUCTION}

Atopic dermatitis (AD) is a chronic inflammatory skin disorder in which patients experience pruritus, erythema, exudation, excoriation, lichenification, and induration/papulation. It occurs in $15-30 \%$ of children and $2-10 \%$ of adults worldwide $[1,2]$. A variety of assessments have been developed and used to evaluate the severity of $\mathrm{AD}$; however, these tools have not been standardized or fully validated [3].

The US Food and Drug Administration recommends the Investigator's (Static) Global Assessment (I[S]GA), which assesses global disease severity using a single-item, multiple construct scale [4], for use in AD. Historically, the ISGA has been subject to modifications and redefinitions when implemented in different trials by different sponsors, leading to a proliferation of ISGA variants in the literature [5]. In general, the ISGA consists of a four- or fivepoint scale, with each score describing an overall body assessment of AD signs/symptoms such as erythema, induration/papulation, lichenification, oozing/crusting, and sometimes excoriation and scaling [6].

The Eczema Area and Severity Index (EASI) is recommended by the Harmonizing Outcome Measures for Eczema initiative and is considered one of the best and most widely used instruments globally to determine AD severity $[3,7,8]$. It assesses the extent and severity of $\mathrm{AD}$ at four body sites (head/neck, trunk, upper extremities, and lower extremities) and measures four clinical signs (erythema, induration/papulation, excoriation, and lichenification), with an overall total score ranging from 0 to 72 [9].

Comparatively, EASI is considered a more clinically objective score than ISGA and has been validated in multiple studies [3], whereas 
only one ISGA scale has been validated to date [10]. Still, studies evaluating AD treatments using both ISGA and EASI have had outcomes that yielded similar response rates (i.e., ISGA success/ISGA clear or almost clear has shown consistent results with EASI-90) [11-15].

Crisaborole ointment, $2 \%$, is a nonsteroidal anti-inflammatory phosphodiesterase 4 inhibitor for the treatment of mild-to-moderate AD. In CORE 1 and CORE 2, two identically designed phase 3 pivotal trials evaluating the efficacy and safety of crisaborole $2 \%$ topical ointment versus vehicle in patients 2 years of age and older with mild-to-moderate $\mathrm{AD}$, the primary outcome was ISGA success (defined as achieving ISGA clear [0] or almost clear [1] with $\mathrm{a} \geq 2$-grade improvement from baseline) at day 29 [2]. EASI data were not collected in these two trials. Although ISGA is recommended by the FDA, EASI is more commonly used worldwide to assess $\mathrm{AD}$ severity; therefore, there was an interest in understanding trial results in terms of EASI scores.

Previous research by Chopra et al. [16] and Leshem et al. [17] evaluated relationships between the ISGA and EASI scales and developed corresponding severity strata for EASI using data from a practice-based prospective study [16] and retrospective analysis [17]. The objective of the present analysis is to apply these published severity strata in a simulation analysis constructed to translate ISGA scores to EASI scores using patient-level data from the clinical trials, thereby aiding in the interpretability of trial data that use ISGA-based endpoints. For this purpose of mapping ISGA to EASI, we used data from the pivotal phase 3 crisaborole trials CORE 1 and CORE 2 that included ISGA scores only. To further interpret the results from our analyses to derive "mapped EASI" scores, we additionally investigated the relationship between changes in ISGA and changes in EASI using data from three studies that included both ISGA and EASI: one phase 2 and two phase 3 trials for the Janus kinase 1 inhibitor abrocitinib.

\section{METHODS}

\section{Study Design for CORE 1 and CORE 2}

The study designs of CORE 1 (NCT02118766) and CORE 2 (NCT02118792) have been published [2]. In brief, these were identically designed, randomized, double-blind, multicenter, vehicle-controlled phase 3 studies examining the efficacy and safety of crisaborole in patients aged $\geq 2$ years with mild-to-moderate $\mathrm{AD}$ as measured by ISGA [2]. Patients were assigned to receive crisaborole or vehicle at a ratio of 2:1 [2]. Treatment was applied to entirely cover all lesions present at baseline (excluding the scalp) twice daily for 28 days [2]. AD severity was assessed at days $1,8,15,22$, and 29 using the ISGA scale (Table 1) [2]. The primary study endpoint was proportion of patients achieving ISGA success, which was defined as clear (0) or almost clear (1) with $a \geq 2$-grade improvement from baseline at day 29. For the purposes of this analysis, data from CORE 1 and CORE 2 were pooled.

An institutional review board at each study site approved the study protocol for the CORE 1 and CORE 2 studies, and written consent was provided by patients or parent(s)/legal guardian(s) [2]. These studies were conducted in compliance with the International Committee on Harmonisation and applicable Good Clinical Practice standards [2] and in accordance with the ethical principles that have their origin in the Declaration of Helsinki.

\section{Mapping of ISGA to EASI}

ISGA scores from CORE 1 and CORE 2 were mapped to EASI scores using published severity strata for EASI from Chopra et al. [16] and Leshem et al. [17]. In Chopra et al., 673 patients with AD aged $13-93$ years (59.6\% female, 59.9\% White) were included in a prospective study [16]. EASI severity strata were based on a fourpoint ISGA index (clear/mild/moderate/severe) (Table 2) [16]. For our analysis, severity strata as defined by Chopra et al. [16] were adapted to create a five-point scale by assigning both the 
Table 1 Definitions for ISGA used as primary endpoint in CORE 1 and CORE 2 [2] (Reprinted from Paller et al. [2], Copyright 2016, with permission from Elsevier)

\begin{tabular}{lll}
\hline Score & Grade & Definition \\
\hline 0 & Clear & Minor residual hypo/hyperpigmentation; no erythema or induration/papulation; no oozing/crusting \\
1 & Almost clear & Trace faint-pink erythema, with barely perceptible induration/papulation and no oozing/crusting \\
2 & Mild & Faint-pink erythema with mild induration/papulation and no oozing/crusting \\
3 & Moderate & Pink-red erythema with moderate induration/papulation with or without oozing/crusting \\
4 & Severe & Deep- or bright-red erythema with severe induration/papulation and with oozing/crusting \\
\hline
\end{tabular}

ISGA Investigator's Static Global Assessment

Table 2 ISGA to EASI severity strata established in Chopra et al. and Leshem et al.

\begin{tabular}{lll}
\hline Study & ISGA & EASI strata \\
\hline Chopra et al. [16] & Clear & 0 \\
& Mild & $0.1-5.9$ \\
& Moderate & $6.0-22.9$ \\
& Severe & $23-72$ \\
Leshem et al. [17] & Clear & 0 \\
& Almost clear & $0.1-1$ \\
& Mild & $1.1-7$ \\
& Moderate & $7.1-21$ \\
& Severe & $21.1-50$ \\
& Very severe & $50.1-72$ \\
\hline
\end{tabular}

EASI Eczema Area and Severity Index, ISGA Investigator's Static Global Assessment

"clear" and "almost clear" categories an EASI score of 0 (Table 3 ).

Leshem et al. was a retrospective study that included 170 patients with $\mathrm{AD}$, of whom $54.1 \%$ were female, $62.4 \%$ were White, and one-third were aged 0-16 years [17]. EASI severity strata proposed by Leshem et al. were based on a sixpoint ISGA index (clear/almost clear/mild/moderate/severe/very severe) (Table 2) [17]. In our analysis, "severe" and "very severe" categories as defined in this study were combined to conform to a five-point ISGA scale (Table 3) [17].

A simulation analysis was performed using the Chopra and Leshem severity strata and pooled data from CORE 1 and CORE 2. Each ISGA score was mapped to a uniform random value of its corresponding EASI strata interval using algorithms derived from Chopra et al. and Leshem et al. (Table 3). Mapping was performed 70,000 times for each ISGA score using SAS version 9.4 (SAS Institute Inc.; Cary, NC, USA).

\section{Transformed Outcomes from CORE 1 and CORE 2}

Generated EASI scores (based on Table 1) were used to provide clinical efficacy measures for trial results from CORE 1 and CORE 2 in every simulation. These outcomes included least squares mean (LSM) percentage change from

Table 3 Adapted EASI severity strata used in simulation analyses

\begin{tabular}{lllllr}
\hline & ISGA 0 & ISGA 1 & ISGA 2 & ISGA 3 & ISGA 4 \\
\hline Adapted Chopra et al. [16] EASI strata & 0 & 0 & $(0.1-5.9)$ & $(6-22.9)$ & $(23-72)$ \\
Adapted Leshem et al. [17] EASI strata & 0 & $(0.1-1)$ & $(1.1-7)$ & $(7.1-21)$ & $(21.1-72)$ \\
\hline
\end{tabular}

EASI Eczema Area and Severity Index, ISGA Investigator's Static Global Assessment 
baseline in EASI and the proportion of patients with 50\%, 75\%, and 90\% improvement (EASI50, EASI-75, and EASI-90, respectively) at day 29 of treatment. These proportions were validated against the studies' primary efficacy endpoint of ISGA success.

\section{Relationship Between Changes in ISGA and Changes in EASI}

To support the validity of the mapping of ISGA to EASI for crisaborole studies, the relationship between changes in ISGA at week 12 and percentage changes in EASI was assessed using data from the following abrocitinib studies that included both IGA and EASI: B7451006 [11], B7451012 (JADE MONO-1) [12], and B7451013 (JADE MONO-2) [13]. EASI percentage changes from baseline at week 12 were rounded to categories ranging from -100 to 100 . ISGA changes from baseline at week 12 were summarized by EASI percentage change categories. In particular, this analysis allowed for an evaluation of whether results from the mapping analyses relative to ISGA response and EASI-90 response (using mapped EASI scores) were consistent with any trends that may be observed when a similar analysis is performed for studies in which both IGA and EASI data were collected directly.

Mean values for IGA change from baseline were plotted against EASI percentage change for the abrocitinib studies. In addition, a simulation was performed for EASI score using 10,000 randomly generated EASI scores (uniform from 0 to 72 ) at baseline and week 12 for the mapping analysis. These scores were then mapped to ISGA scores using an inversion of Leshem et al. mapping. Simulated ISGA changes from baseline at week 12 were then summarized by EASI percentage change categories and assessed in conjunction with the three studies.

\section{RESULTS}

\section{Simulation Analysis}

The pooled study population used in this analysis consisted of 1016 patients treated with crisaborole and 506 patients treated with vehicle. In total, 70,000 simulations were performed for each of the EASI severity strata classifications published by Chopra et al. [16] and Leshem et al. [17]. The LSM (standard error) percentage change from baseline in simulated EASI scores at day 29 (crisaborole versus vehicle) was $-26.3 \%$ (17) versus $45.2 \%$ (35) using the Chopra strata (difference, $-71.5 ; 95 \%$ CI -147.0 to $5.0 ; P=$ $0.0671)$ and $-43.1 \%$ (4.6) versus $-5.2 \%(8.4)$ using the Leshem strata (difference, $-37.8 ; 95 \%$ CI -56.2 to $-19.5 ; P<0.0001)$. Distribution of simulation results can be found in Supplementary Fig. S1.

Using the adapted Chopra strata, EASI-50 at day 29 (crisaborole versus vehicle) from simulation analysis was $72.1 \%$ versus $57.6 \%$ $(P<0.0001)$ (Fig. 1a). Similarly, EASI-50 at day 29 (crisaborole versus vehicle) was $68.8 \%$ versus $54.0 \%$ using the adapted Leshem strata $(P<0.0001)$ (Fig. 1a). For EASI-75, response rates at day 29 were $63.0 \%$ versus $47.8 \%$ using the Chopra strata $(P<0.0001)$ and $54.8 \%$ versus $40.5 \%$ using the Leshem strata $(P<0.0001)$ (Fig. 1b). EASI-90 rates at day 29 were $55.0 \%$ versus $40.1 \%$ with the Chopra strata $(P<0.0001)$ and $38.9 \%$ versus $27.2 \%$ with the Leshem strata $(P<0.0001)$ (Fig. 1c). Distribution of simulation results for EASI-50, EASI-75, and EASI-90 are shown in Supplementary Figs. S2, S3, and S4.

In separate analyses of severity outcomes from three abrocitinib studies that included ISGA scores and EASI scores for assessment of $\mathrm{AD}$ severity, the relationship between changes in ISGA following 12 weeks of treatment and percentage change in EASI was evaluated. These analyses showed that a mean two-point improvement in ISGA was comparable to a $90 \%$ improvement in EASI (Fig. 2). 

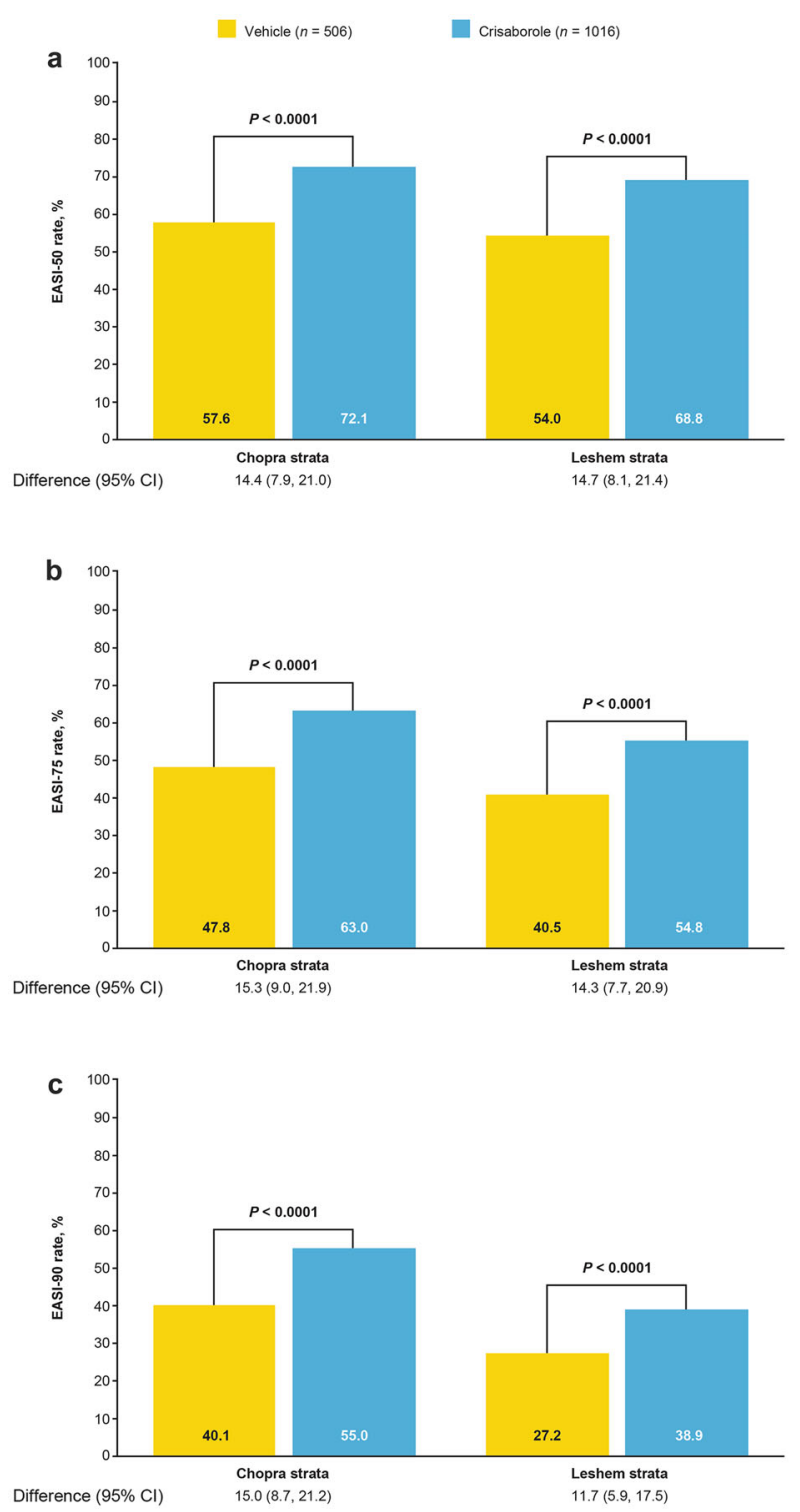

Fig. 1 Generated day 29 outcomes for a EASI-50, b EASI-75, and c EASI-90. EASI Eczema Area and Severity Index

\section{DISCUSSION}

To the best of the authors' knowledge, the analysis presented here is the first known mapping of ISGA scores to EASI scores using clinical trial data. Overall, the translation of ISGA scores from pooled data from CORE 1 and CORE 2 to EASI scores using the two severity strata classifications showed consistent results. All of the clinical outcome measures using either Chopra or Leshem severity strata for computation of mapped EASI demonstrated differences between crisaborole and vehicle that were statistically significant, except for percentage change from baseline in EASI score using the Chopra strata $(P=0.0671)$. Because ISGA $=1$ was mapped to 0 using the algorithm derived from Chopra et al., the mapped EASI percentage change from baseline had high variability.

Mapped EASI results were consistent with findings for ISGA in the CORE 1 and CORE 2 trials [2]. Superiority of crisaborole to vehicle as assessed by ISGA success and ISGA clear or almost clear [2] was maintained in mapped EASI 


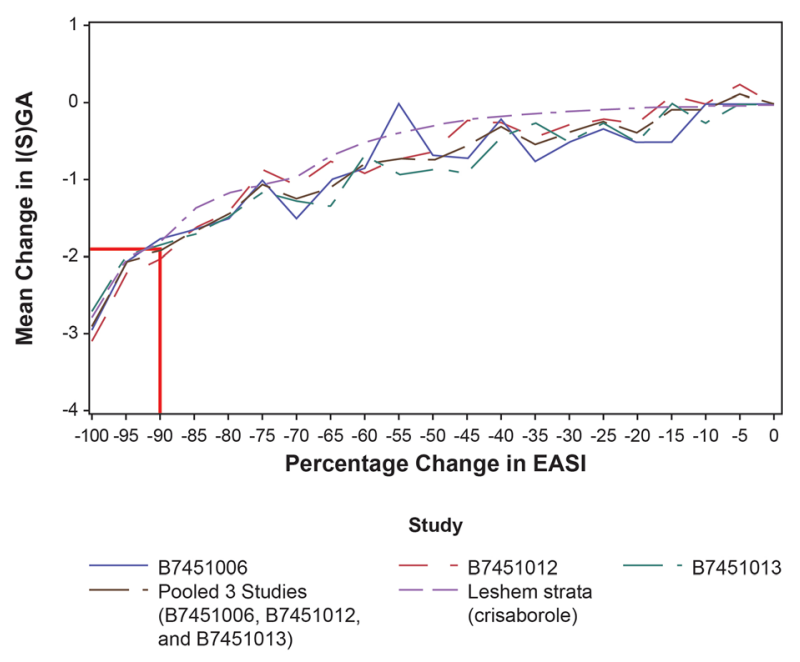

Fig. 2 Mean change in $\mathrm{I}(\mathrm{S})$ GA by percentage change in EASI at week 12. EASI Eczema Area and Severity Index, $I(S) G A$ Investigator's (Static) Global Assessment. Intersection of the red lines indicates that a mean two-point improvement in $\mathrm{I}(\mathrm{S}) \mathrm{GA}$ corresponds to $90 \%$ improvement in EASI

scores, and rates of ISGA success (crisaborole versus vehicle) in both trials (CORE 1: $32.8 \%$ versus 25.4\%; CORE 2: $31.4 \%$ versus $18.0 \%$ ) were comparable to the rates of EASI-90 response that were mapped in this analysis.

It has been observed that although the ISGA and EASI are very different clinical assessment measures with respect to construct (e.g., content and level of detail), consistent efficacy results have been observed when each of these two clinical measures of severity have been used in the same study in multiple clinical trials of patients with AD [11-15]. ISGA response rates were consistently similar to EASI-90 response rates in one phase 2 and two phase 3 trials of abrocitinib in patients with moderate-to-severe AD [11-13]. For example, IGA response rates (defined as IGA score of 0 or 1 and reduction of $\geq 2$ points from baseline) at week 12 in the JADE MONO-1 study were $44 \%, 24 \%$, and $8 \%$ for abrocitinib $200 \mathrm{mg}$ and $100 \mathrm{mg}$ and placebo groups, respectively, in one phase 3 study, whereas EASI-90 response rates were 39\%, 19\%, and $5 \%$ in each group, respectively, in this same study [12]. In the phase 3 SOLO 1 and SOLO 2 trials of the anti-interleukin 4 receptor $\alpha$ monoclonal antibody dupilumab in patients with moderate-to-severe $\mathrm{AD}$, week 16 IGA responses were approximately $36-38 \%$ in the dupilumab arms compared with $8-10 \%$ in the placebo arms [14]. EASI-90 response rates were similar, with response rates of $30-36 \%$ in the dupilumab arms and $7-8 \%$ in the placebo arms [14]. These results are consistent with our findings for crisaborole given that a two-point improvement in ISGA has been shown in our analyses of 3 relevant abrocitinib studies to correspond to a $90 \%$ improvement in EASI. These results support the validity of the estimated treatment effects from the CORE 1 and CORE 2 studies represented in this analysis in terms of EASI.

\section{Limitations}

There were several limitations for this analysis, including the fact that most patients in the publications by Chopra et al. [16] and Leshem et al. [17], on which our severity strata to translate ISGA to EASI were based, were adults, whereas the CORE 1 and CORE 2 populations used in our analysis included mostly pediatric patients $\geq 2$ years of age. There were also inherent limitations in translating ISGA scores to EASI scores because we were required to transition from a more limited five-point ordinal scale to a continuous scale ranging from 0 to 72. Lastly, the outcome of ISGA success (clear or almost clear with $\mathrm{a} \geq 2$-grade improvement) is a stringent endpoint and is clinically difficult to achieve for patients with mild-to-moderate $\mathrm{AD}$; this limitation is also relevant for subsequently mapped EASI endpoints.

\section{CONCLUSIONS}

In this analysis, the mapping of ISGA to EASI scores was investigated using data from clinical trials to present results in terms of mapped EASI scores, thereby aiding in interpretability of ISGA for various stakeholders (patients, physicians and other providers, regulatory and/or reimbursement authorities) involved in making health care decisions. This analysis expands on past severity strata evaluations for EASI to provide an approach for mapping ISGA to EASI scores using clinical trial data. It shows the 
consistency of results obtained using mapped EASI scores when applying simulation methodologies across clinical outcomes measures, an approach that may be useful for assisting in interpretability of the ISGA outcomes reported in other clinical studies. Ongoing studies evaluating crisaborole that include ISGA- and EASI-based outcomes will provide data sources for further validating our results from these simulation analyses.

\section{ACKNOWLEDGEMENTS}

Funding. This study and the journal's Rapid Service fee were funded by Pfizer Inc.

Medical Writing, Editorial, and Other Assistance. Editorial/medical writing support under the guidance of the authors was provided by Stephanie Agbu, PhD, and Robert J. Schoen, PharmD, at ApotheCom, San Francisco, CA, USA, and was sponsored by Pfizer Inc., New York, NY, USA, in accordance with Good Publication Practice (GPP3) guidelines (Ann Intern Med. 2015;163:461-464).

Authorship. All named authors meet the International Committee of Medical Journal Editors (ICMJE) criteria for authorship for this article, take responsibility for the integrity of the work as a whole, and have given their approval for this version to be published.

Prior Presentation. This analysis was previously presented at the International Society for Pharmacoeconomics and Outcomes ResearchEurope 2020 meeting, November 16-19, 2020, and the 3rd Annual Revolutionizing Atopic Dermatitis conference, December 13-14, 2020. Both congresses were held virtually.

Disclosures. Jacob P. Thyssen is an advisor/ investigator or speaker for AbbVie, Eli Lilly, LEO Pharma, Regeneron, and Sanofi-Genzyme. Chuanbo Zang, Maureen P. Neary, Andrew G. Bushmakin, Joseph C. Cappelleri, Amy Cha, and Christopher Russo are employees and stockholders of Pfizer Inc. Thomas A. Luger is an investigator for AbbVie, Celgene, Eli Lilly, LEO Pharma, Menlo Therapeutics, Novartis, Pfizer, and Sandoz; is a member of scientific advisory boards for AbbVie, Argenx, Celgene, Ceres Pharma, Eli Lilly, Galderma, Janssen-Cilag, La Roche-Posay, LEO Pharma, Menlo Therapeutics, Mylan/Meda AB, Novartis, Pfizer, Pierre Fabre, Piqur Therapeutics, Sandoz, Sanofi-Aventis, and Symrise; and has received funding from AbbVie, Celgene, Janssen-Cilag, Merck Sharp \& Dohme, Mylan/Meda AB, Novartis, Pfizer, and Wolff Laboratories.

Compliance with Ethics Guidelines. An institutional review board at each study site approved the study protocol for the CORE 1 and CORE 2 studies, and written consent was provided by patients or parent(s)/legal guardians. These studies were conducted in compliance with the International Committee on Harmonisation (ICH) and applicable Good Clinical Practice (GCP) standards and in accordance with the ethical principles that have their origin in the Declaration of Helsinki.

Data Availability. Upon request, and subject to certain criteria, conditions, and exceptions (see https://www.pfizer.com/science/ clinical-trials/trial-data-and-results for more information), Pfizer will provide access to individual deidentified participant data from Pfizersponsored global interventional clinical studies conducted for medicines, vaccines, and medical devices (1) for indications that have been approved in the United States and/or EU or (2) in programs that have been terminated (ie, development for all indications has been discontinued). Pfizer will also consider requests for the protocol, data dictionary, and statistical analysis plan. Data may be requested from Pfizer trials 24 months after trial completion. The deidentified participant data will be made available to researchers whose proposals meet the research criteria and other conditions, and for which an exception does not apply, via a secure portal. To gain access, data requestors must enter into a data access agreement with Pfizer. 
Open Access. This article is licensed under a Creative Commons Attribution-NonCommercial 4.0 International License, which permits any non-commercial use, sharing, adaptation, distribution and reproduction in any medium or format, as long as you give appropriate credit to the original author(s) and the source, provide a link to the Creative Commons licence, and indicate if changes were made. The images or other third party material in this article are included in the article's Creative Commons licence, unless indicated otherwise in a credit line to the material. If material is not included in the article's Creative Commons licence and your intended use is not permitted by statutory regulation or exceeds the permitted use, you will need to obtain permission directly from the copyright holder. To view a copy of this licence, visit http://creativecommons.org/licenses/by$\mathrm{nc} / 4.0 /$.

\section{REFERENCES}

1. Bieber T. Atopic dermatitis. $\mathrm{N}$ Engl $\mathrm{J}$ Med. 2008;358(14):1483-94.

2. Paller AS, Tom WL, Lebwohl MG, et al. Efficacy and safety of crisaborole ointment, a novel, nonsteroidal phosphodiesterase 4 (PDE4) inhibitor for the topical treatment of atopic dermatitis $(\mathrm{AD})$ in children and adults. J Am Acad Dermatol. 2016;75(3):494-503.e6.

3. Schmitt J, Langan S, Williams HC, European D-E. What are the best outcome measurements for atopic eczema? A systematic review. J Allergy Clin Immunol. 2007;120(6):1389-98.

4. US Food and Drug Administration. Draft guidance on pimecrolimus. 2012. https://www.accessdata.fda. gov/drugsatfda_docs/psg/PSG_021302.pdf. Accessed 19 Feb 2021.

5. Barrett A, Hahn-Pedersen J, Kragh N, Evans E, Gnanasakthy A. Patient-reported outcome measures in atopic dermatitis and chronic hand eczema in adults. Patient. 2019;12(5):445-59.

6. Futamura M, Leshem YA, Thomas KS, Nankervis H, Williams HC, Simpson EL. A systematic review of Investigator Global Assessment (IGA) in atopic dermatitis (AD) trials: many options, no standards. J Am Acad Dermatol. 2016;74(2):288-94.
7. Schmitt J, Langan S, Deckert S, et al. Assessment of clinical signs of atopic dermatitis: a systematic review and recommendation. J Allergy Clin Immunol. 2013;132(6):1337-47.

8. Schmitt J, Spuls PI, Thomas KS, et al. The Harmonising Outcome Measures for Eczema (HOME) statement to assess clinical signs of atopic eczema in trials. J Allergy Clin Immunol. 2014;134(4):800-7.

9. Hanifin JM, Thurston M, Omoto M, Cherill R, Tofte SJ, Graeber M. The eczema area and severity index (EASI): assessment of reliability in atopic dermatitis. EASI Evaluator Group. Exp Dermatol. 2001;10(1):11-8.

10. Eli Lilly and Company. Validated investigator global assessment scale for atopic dermatitis. 2017. https://www.eczemacouncil.org/assets/docs/Valida ted-Investigator-Global-Assessment-Scale_vIGA-AD_ 2017.pdf. Accessed 19 Feb 2021.

11. Gooderham MJ, Forman SB, Bissonnette R, et al. Efficacy and safety of oral Janus kinase 1 inhibitor abrocitinib for patients with atopic dermatitis: a phase 2 randomized clinical trial. JAMA Dermatol. 2019;155(12):1371-9.

12. Simpson EL, Sinclair R, Forman S, et al. Efficacy and safety of abrocitinib in adults and adolescents with moderate-to-severe atopic dermatitis (JADE MONO1): a multicentre, double-blind, randomised, placebo-controlled, phase 3 trial. Lancet. 2020;396(10246):255-66.

13. Silverberg JI, Simpson EL, Thyssen JP, et al. Efficacy and safety of abrocitinib in patients with moderateto-severe atopic dermatitis: a randomized clinical trial. JAMA Dermatol. 2020;156(8):863-73.

14. Simpson EL, Bieber T, Guttman-Yassky E, et al. Two phase 3 trials of dupilumab versus placebo in atopic dermatitis. N Engl J Med. 2016;375(24):2335-48.

15. Guttman-Yassky E, Thaci D, Pangan AL, et al. Upadacitinib in adults with moderate to severe atopic dermatitis: 16-week results from a randomized, placebo-controlled trial. J Allergy Clin Immunol. 2020;3:877-84.

16. Chopra R, Vakharia PP, Sacotte R, et al. Severity strata for Eczema Area and Severity Index (EASI), modified EASI, Scoring Atopic Dermatitis (SCORAD), objective SCORAD, Atopic Dermatitis Severity Index and body surface area in adolescents and adults with atopic dermatitis. Br J Dermatol. 2017;177(5):1316-21.

17. Leshem YA, Hajar T, Hanifin JM, Simpson EL. What the Eczema Area and Severity Index score tells us about the severity of atopic dermatitis: an interpretability study. Br J Dermatol. 2015;172(5): 1353-7. 